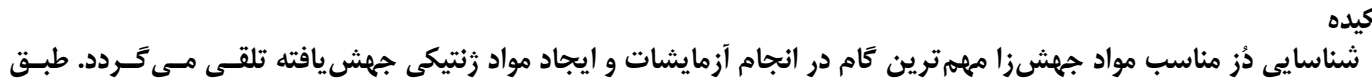

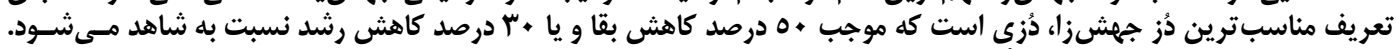

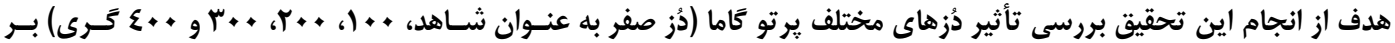

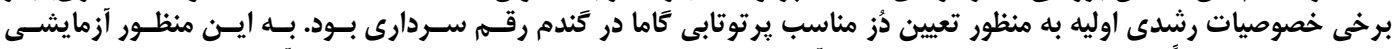

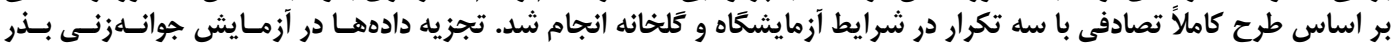

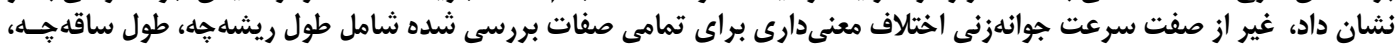

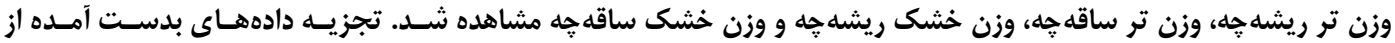

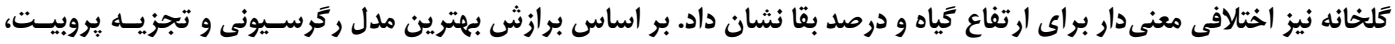

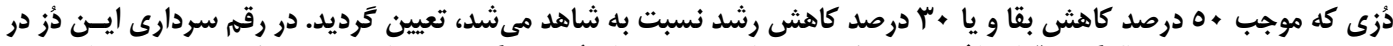

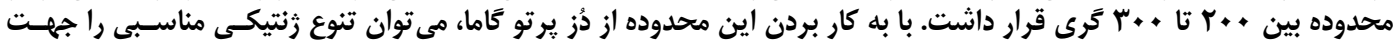

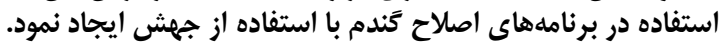

وازههاى كليدى: اصلاح موتاسيونى، كبالت + 7، مدل رتخرسيونى، تنوع زنتيكى، كَندم

يرتوتابى براى القا جهش مورد ارزيابى قـرار مـى گيرنسد، اكثـر

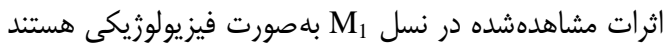

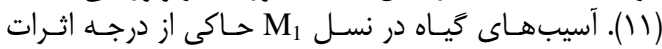

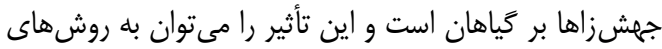

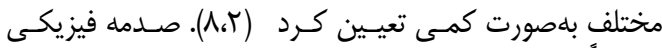

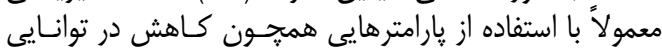

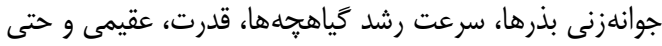

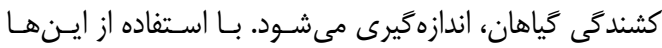

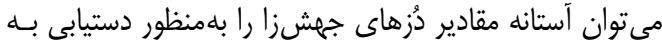

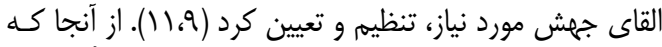

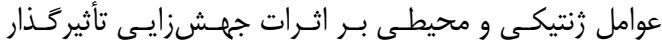

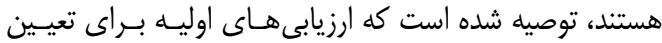

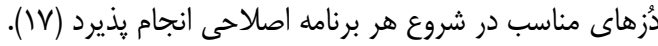

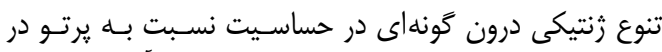

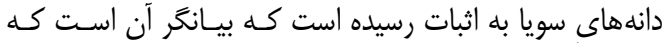

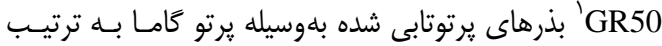

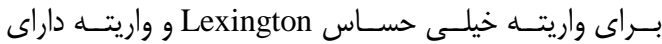

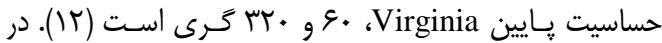

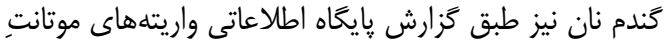

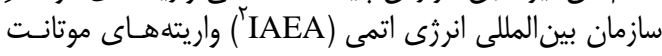

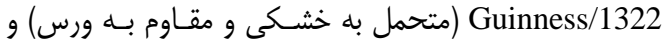

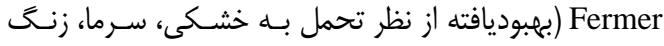

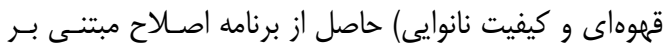

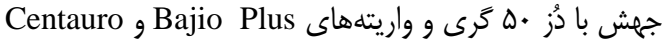

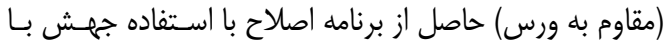

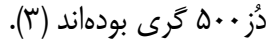

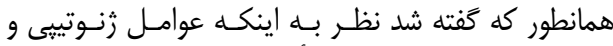

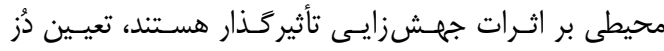

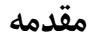

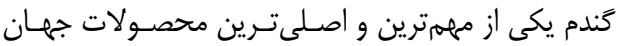

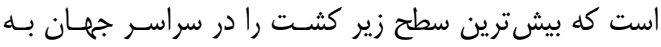

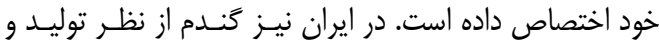

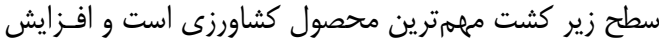

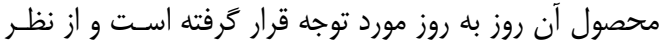

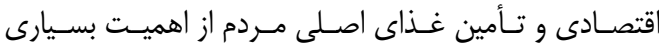

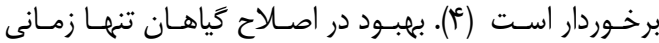

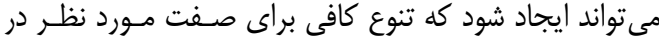

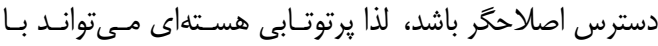

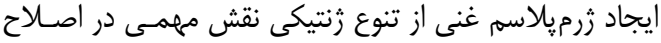

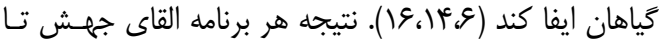

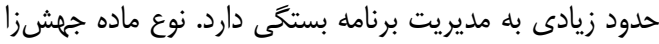

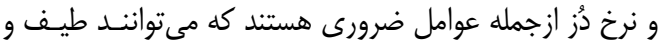

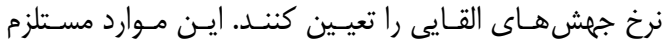

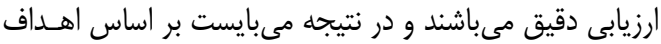

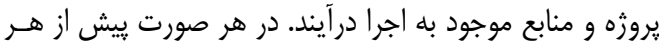

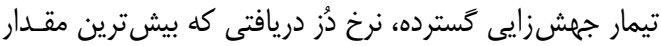

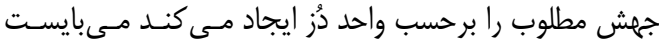

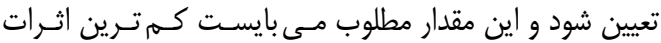

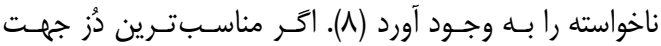

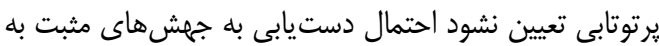

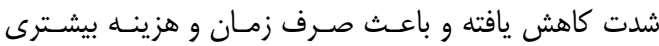

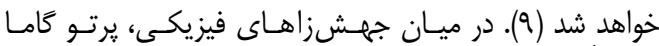

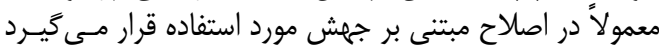

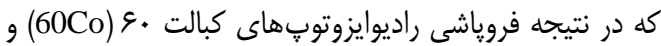

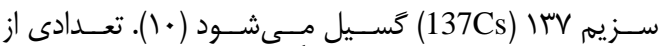

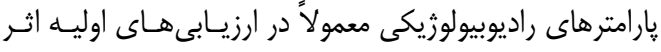




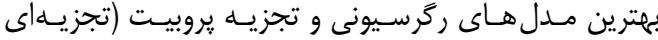

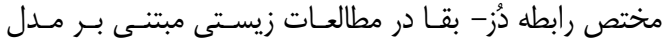

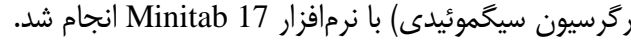

تجزيه واريانس دادهها در آزمايش جوانهزنى نشان داد غير إنير

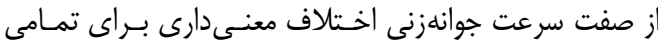

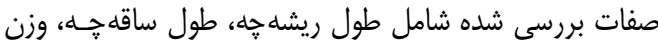

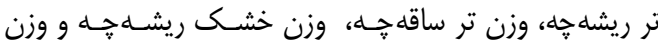

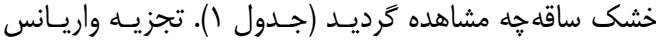

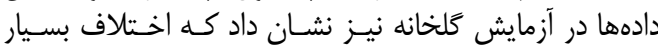

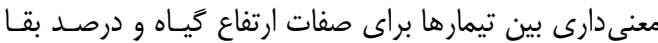

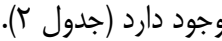

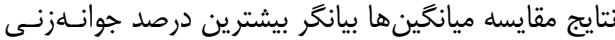

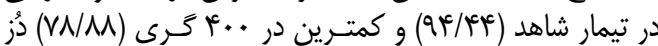

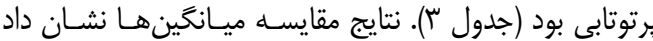

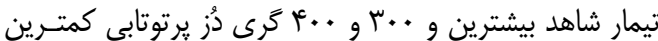

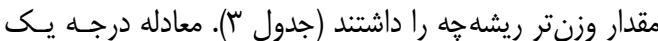

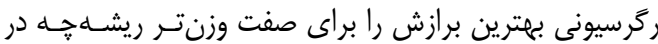

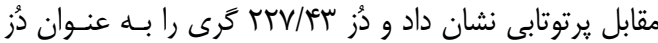

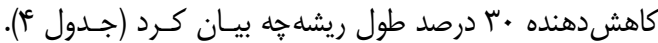

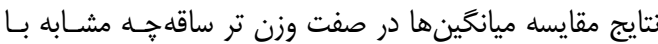

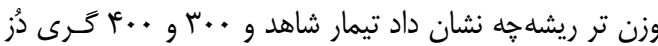

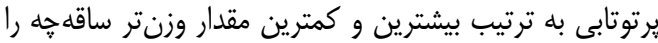

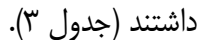

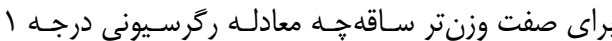

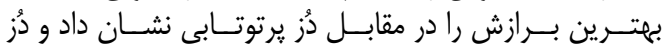

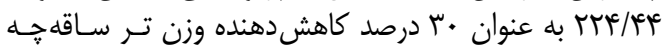

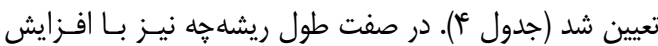

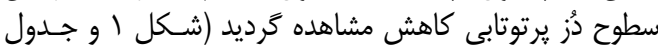

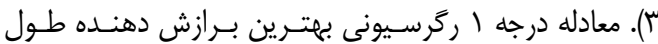

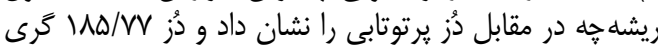

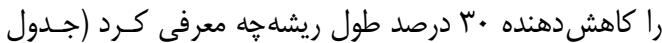

مناسب برتوتابى در آغاز يك برنامه اصلاح با استفاده از جهش

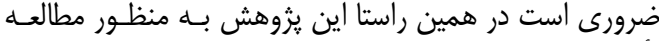

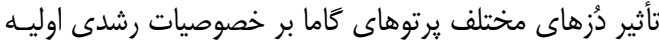

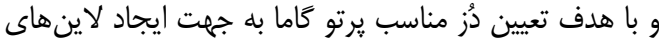
جهش يافته مطلوب در رقمم كَندم نان سردارى به به انجام رسيد.

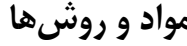

نحوه تيماردهى بذرها

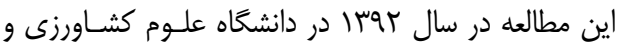

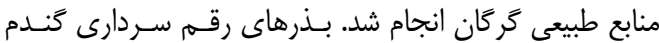

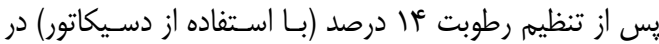

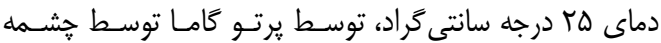

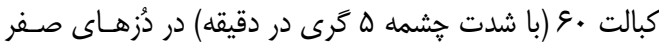

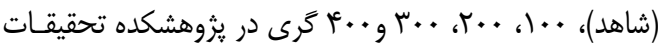

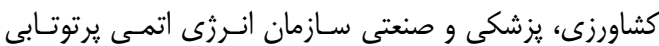

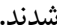

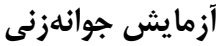

اين آزمايش در قالب طرح كاملاً تصادفى با سه تكرار اجرا

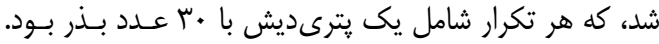

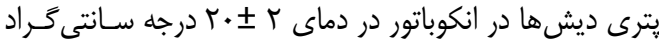

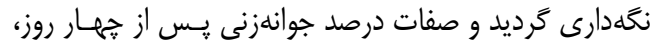

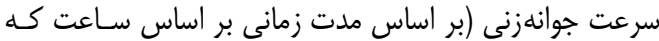

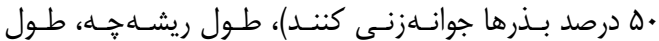

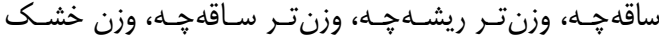
ريشه קه و وزن خشك ساقه آزمايش كَلخانه آزمايش در قالب طرح كاملهاً تصادفى با سه تكرار به به اجـرا

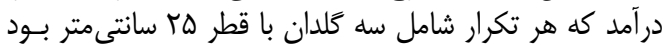

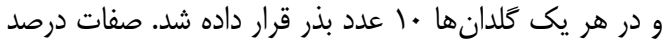

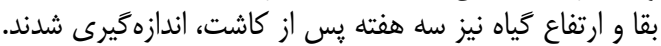
تجزيه و تحليل آمارى

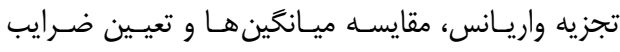
همبستخى ساده با استفاده از نـرم|فزار 9.1 .3 SAS و تعيـين

Table 1. Analysis of variance for traits in germination experiment

جدول ا- تجزيه واريانس صفات در آزمايش جوانهزنى

\begin{tabular}{|c|c|c|c|c|c|c|c|c|c|}
\hline \multicolumn{8}{|c|}{ ميانكين مربعات } & \multirow[b]{2}{*}{ df } & \multirow[b]{2}{*}{ منابع تغيير } \\
\hline وزاقه خشه & وزيش خشهى & ساقه ته & وزيشن تر & ساقهول & ريشه جه & سرعت جوانهزنى & جوانهزني & & \\
\hline.$/ . .9^{* * * i n}$ & $.1 . \cdot 19^{* * a n}$ & $\cdot / / V^{* * a}$ & $\cdot / K F Y^{\prime}$ & $1 / 89 r^{*}$ & $1 / \Lambda \Delta V^{\text {sets }}$ & $\cdot(\cdots \cdot \cdot)^{f^{\mathrm{ns}}}$ & 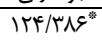 & r & تيمار (دُز) \\
\hline.$/ \cdots v$ & $\cdot \cdots \cdots \Delta$ &...$\mu \Delta$ & 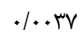 & $\cdot / M T I$ & .1 .09 & gr.... & $r \Delta / \Lambda \cdot \Gamma$ & 1. & خطا \\
\hline $11 / 90$ & IT/GK & $g / r V$ & $\mid 1 / \pi$ & $g / r V$ & $|r / V|$ & $9 / \mathrm{VV}$ & $\Delta / V Q$ & & ضريب تغيير \\
\hline
\end{tabular}

Table 2. Analysis of variance for traits in greenhouse experiment

جدول r- تجزيه واريانس صفات در آزمايش كلخانه

\begin{tabular}{|c|c|c|c|}
\hline \multicolumn{2}{|c|}{ ميانگين مربعات } & \multirow{2}{*}{$\mathrm{df}$} & \multirow{2}{*}{ منابع تغيير } \\
\hline درصد بقا & ارتفاع & & \\
\hline$T V \Delta S / \& 9 S^{* *}$ & 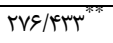 & F & تيمار (دُز) \\
\hline זسז//צר & $r / 1 Q$ & 1. & خطا \\
\hline $10 / \cdot 9$ & $9 / T V$ & & ضريب تغيير \\
\hline
\end{tabular}


Table 3. Mean comparison of measured traits

جدول س- مقايسه ميانخين صفات اندازهيرى شده

\begin{tabular}{|c|c|c|c|c|c|c|c|c|c|}
\hline $\begin{array}{l}\text { بقا } \\
\text { (\%) }\end{array}$ & $\begin{array}{l}\text { ارتفاع } \\
\text { (cm) }\end{array}$ & ساقهن خشه (g) & $\begin{array}{c}\text { وريش خشهه } \\
\text { (g) } \\
\text { (g) }\end{array}$ & وزن ترساقه & ريشه קهن (g) & $\begin{array}{c}\text { طول ساقه } \\
\text { (cm) }\end{array}$ & $\begin{array}{l}\text { طول ريشه }(\mathrm{cm}) \\
\text { (cm) }\end{array}$ & $\begin{array}{l}\text { جوانهزنى (\%) } \\
\text { (\% }\end{array}$ & $\begin{array}{c}\text { (Gy) } \\
\text { (Gy) }\end{array}$ \\
\hline 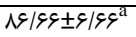 & $r \mathcal{R} / \Gamma^{\prime} \pm 1 / \Delta^{\mathrm{a}}$ & $. / 119 \pm .1 \cdot .9^{a}$ & $\cdot / \cdot 11 \pm \cdot / \cdot \cdot r^{a}$ & $T / T V \pm \cdot / \cdot r^{a}$ & $\cdot / 9 \& \pm \cdot / \cdot \Delta^{a}$ & $r / \varepsilon \Delta \pm \cdot / \backslash \Delta^{a}$ & 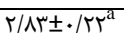 & $q \psi / 4 \psi \pm \psi / \cdot .^{a}$ & (شاهد) \\
\hline$q . / \cdot \pm \Delta / v v^{a}$ & $r q / . . \pm . / 9^{a}$ & $. / .99 \pm . / . \cdot r^{\mathrm{b}}$ & $.1 .9 . \pm .1 . .9^{\mathrm{b}}$ & $1 / \cdot \Delta \pm \cdot / \cdot r^{b}$ & $\cdot \mid q \wedge \pm \cdot / \cdot r^{\mathrm{D}}$ & $r / r \cdot \pm \cdot / 11^{b}$ & $r / q \mu \pm . / / f^{\mathrm{a}}$ & $9 r / r r \pm 1 / 11^{\text {ab }}$ & $1 .$. \\
\hline$\Lambda \cdot 199 \pm \Delta / \mathrm{V}^{\mathrm{a}}$ & r سr/ & $. / .9 \Delta \pm . / . . r^{D}$ & $. / . r \Lambda \pm . / . . l^{c}$ & $\cdot / 91 \pm \cdot / \cdot \Delta^{\mathrm{C}}$ & $. / \Leftarrow q \pm \cdot / \cdot r^{\mathrm{c}}$ & $r / \cdot \Delta \pm \cdot / \cdot \varphi^{0}$ & I/VA $\pm \cdot / / \mathrm{T}^{\mathrm{D}}$ & 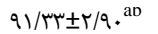 & $r .$. \\
\hline$\tau \cdot / \cdots \pm \Delta / V V^{\mathrm{D}}$ & $19 / . . \pm r / r r^{D}$ & $. / \cdot r \Psi \pm \cdot / . r^{c}$ & $. / \cdot r t \pm . / . \cdot r^{c}$ & $\cdot / V^{e \pm \pm \cdot / \cdot r^{d}}$ & / & $r / 4 \mid \pm \cdot / \cdot \Lambda^{c}$ & $1 / 19 \pm \cdot / .9^{c}$ & $\Lambda F / \epsilon F \pm r / q \Psi^{b c}$ & r.. \\
\hline סسץ/سזעس & $\Gamma / \mathcal{V} \pm . / M^{c}$ & $. / \cdot 1 r \pm \cdot / . .1^{\mathrm{a}}$ & $\cdot / \cdot|v \pm \cdot / \cdot \cdot|^{\mathrm{a}}$ & $\cdot 198 \pm \cdot / \cdot r^{\mathrm{d}}$ & $\cdot / r \Delta \pm \cdot / \cdot r^{\mathrm{d}}$ & $1 / V r \pm \cdot / \cdot 9^{d}$ & $1 / / \varepsilon \pm \cdot / \cdot \Delta^{c}$ & $V N / M \pm r / q \mu^{c}$ & $f .$. \\
\hline
\end{tabular}

Table 4. Proper equation that describes 30\% reduction in different traits

جدول ع- معادله مناسب توصيف كننده .r \% كاهش در صفات مختلف

\begin{tabular}{|c|c|c|c|}
\hline ضريب تبيين & معادله & 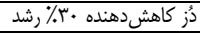 & صفت \\
\hline $91 / V$ & 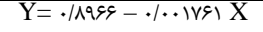 & $T T V / F T$ & وزن تر ريشه هـ \\
\hline $9 T / 9$ & $\mathrm{Y}=1 / r r \Delta-\cdot / \cdot \cdot \Delta \overrightarrow{\mid} \cdot \mathrm{X}$ & TME/AY & وزن تر ساقه \\
\hline$\Lambda F / V$ & $\mathrm{Y}=r / \Lambda V V-\cdot / \cdot$ rVqr $\mathrm{X}$ & $1 N \Delta / V V$ & طول ريشهحه \\
\hline $9 \cdot / 0$ & $\mathrm{Y}=r / v r q-\cdot / \cdot r q \mu \Delta \mathrm{X}$ & $r \Delta F / \Delta D$ & طول ساقه حه \\
\hline$\wedge \mathrm{V} / \cdot$ & $\mathrm{Y}=\cdot / 1 r \Delta \Lambda-\cdot / \cdots r V r \mathrm{X}$ & TET/TY & وزن خشك ساقه \\
\hline $91 / \pi$ & 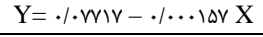 & rTq/II & وزن خشك ريشه \\
\hline
\end{tabular}

براى وزن خشك ريشه خه در مقابل دُز يرتوتابى معادله درجـهـ

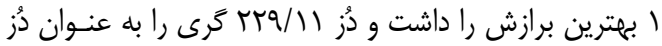

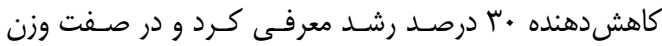

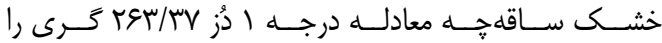

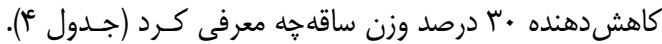

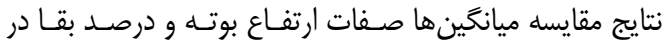

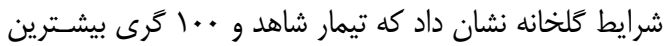

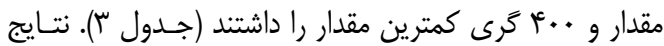

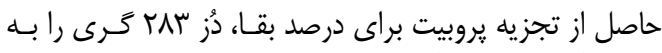

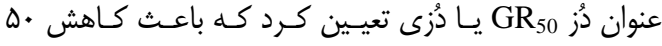

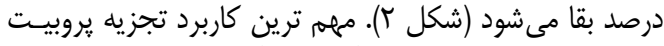

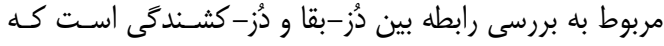

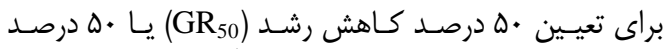

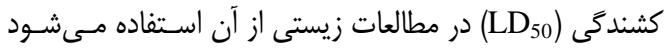

(10)

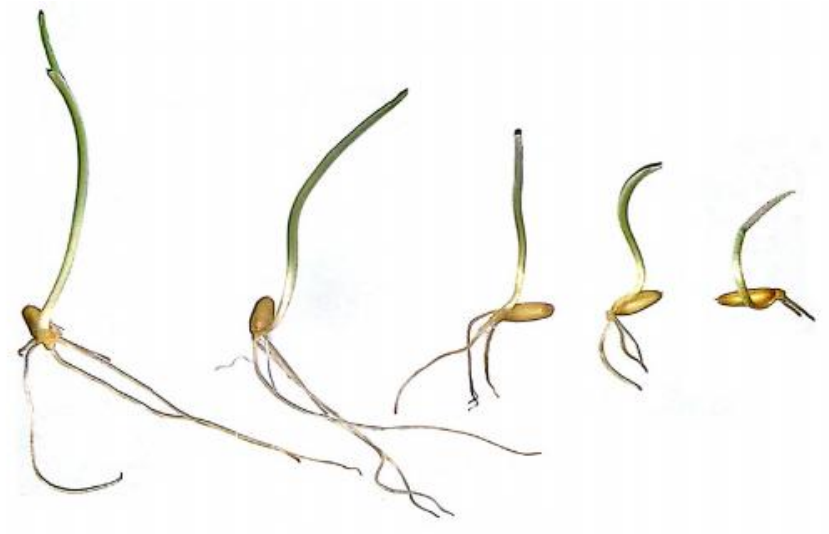

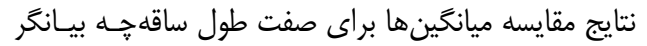

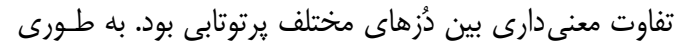

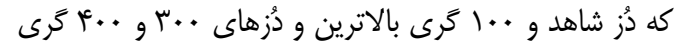

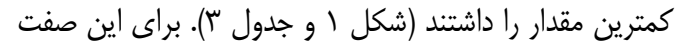

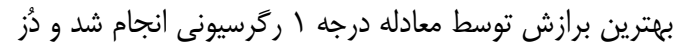

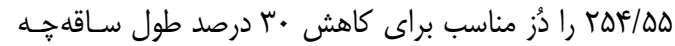

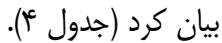

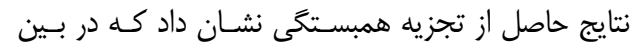

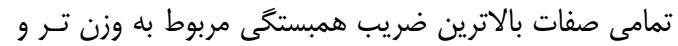

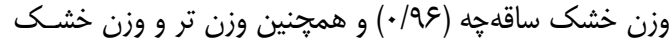

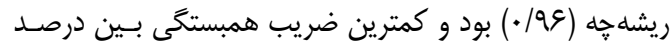

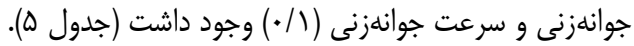

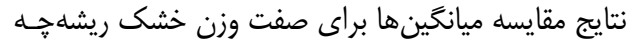

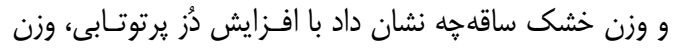
خشك بلهور معنى دارى كاهش مى خيابد (جدول بَ).

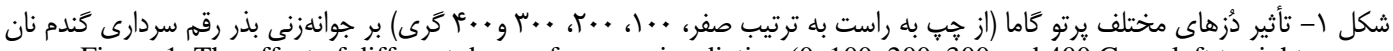
Figure 1. The effect of different doses of gamma irradiation (0, 100, 200, 300 and 400 Gary. left to right, respectively) on seed germination of Sardari bread wheat 
Table 5. Simple correlation coefficients between measured traits

جدول هـ ضرايب همبستخى ساده بين صفات اندازهگيرى شده

\begin{tabular}{|c|c|c|c|c|c|c|c|c|c|}
\hline & ارتفاع & طول ريشه & طول ساقه קه & روزيشن تر & ساقه ته & وزيشه خه & وزاقه خشه & جوانهزنى درص & جوانهزعنى \\
\hline ارتفاع & 1 & & & & & & & & \\
\hline درصد بقا &.$|9 T|^{-\pi T}$ & & & & & & & & \\
\hline طول ريشه & $\cdot / V G V^{m-T}$ & 1 & & & & & & & \\
\hline طول ساقه קه" & . & $\cdot / \Lambda \Delta \Lambda^{\pi *}$ & 1 & & & & & & \\
\hline وزن تر ريشهجه & $\cdot / V V g^{\pi T}$ &.$/ 919^{* \pi}$ & $\cdot / M M^{\pi *}$ & 1 & & & & & \\
\hline وزن تر ساقهاه & $\left.\cdot|\Lambda|\right|^{m \pi}$ &.$/ 194 \Lambda^{-1 \pi}$ &.$/ 91 f^{-m}$ &.$/ 9 \Delta F^{N \pi}$ & 1 & & & & \\
\hline وزن خشه & . /var ${ }^{* * *}$ & $\cdot / 9 \cdot r^{* *}$ & $\cdot / M q^{* *}$ &.$/\left.99\right|^{* * *}$ &.$/ 99 \Lambda^{* *}$ & 1 & & & \\
\hline وزاقه خشه & . $/ 9 .^{* * *}$ & $\cdot|1| 9^{* *}$ & . $/ 9 \pi r^{* *}$ & $\cdot / \Lambda \Delta \Gamma^{* * *}$ & $\cdot / 9 \cdot 0^{* *}$ & $\cdot / \Lambda q^{* * *}$ & 1 & & \\
\hline درصد جوانهزنىى & $\cdot / V r^{-\pi T}$ & $\cdot / V \Delta S^{N T}$ & $\cdot / \Lambda \cdot r^{m \pi}$ & $\cdot / V T V^{\pi-\pi}$ & $\cdot / V T \cdot{ }^{* T}$ & $.19 \mathrm{Vr}^{\pi \omega}$ &.$/ V q^{* *}$ & 1 & \\
\hline سرعت جوانهزنى & $\cdot|\& \Delta|$ & . & •/rqv &.$/ F T \Lambda$ & - TrAF & . & - Tat & .11 .9 & 1 \\
\hline
\end{tabular}

بود كـهـ نشـان دهنــده تـأثير يـايين ايـن دو عامـل در شـرايط

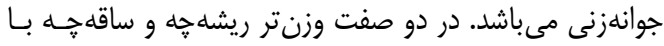

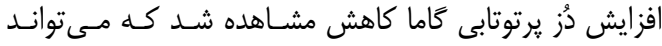

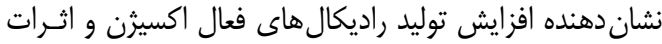

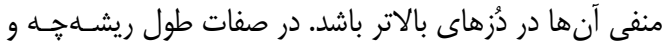

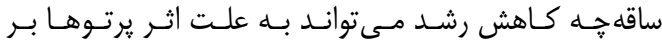

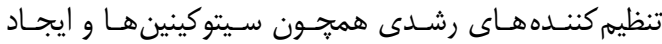

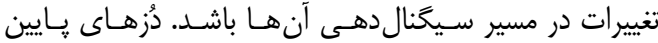

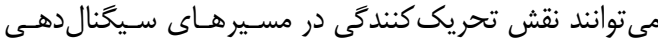

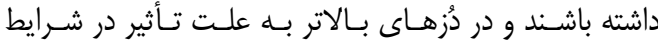

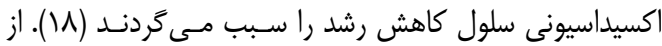

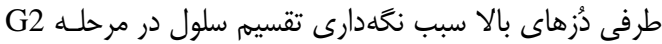

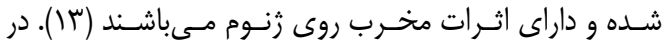

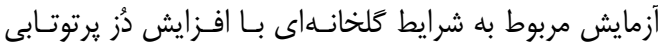

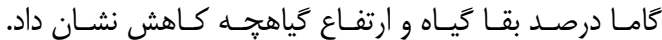

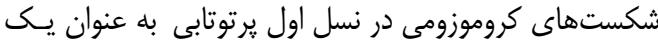

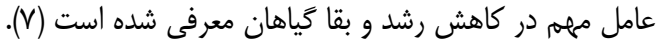

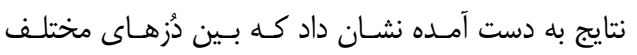

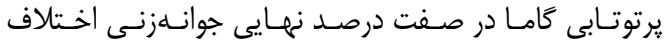

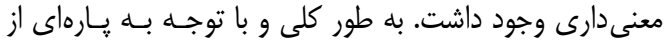

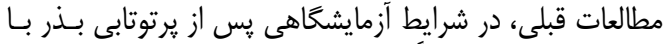

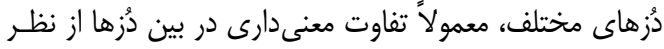

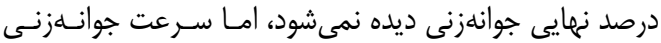

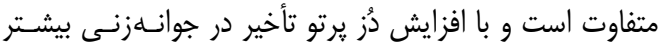

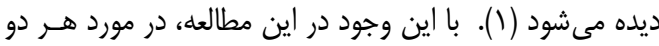

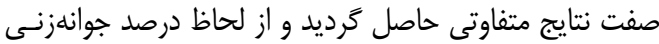

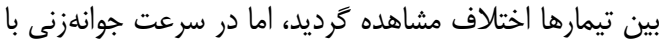

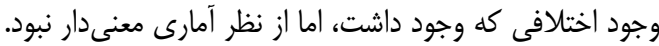

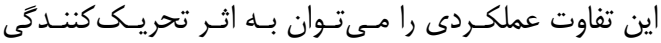

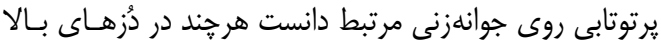

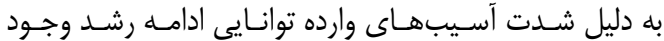

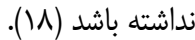

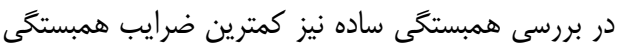

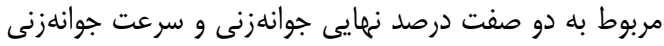

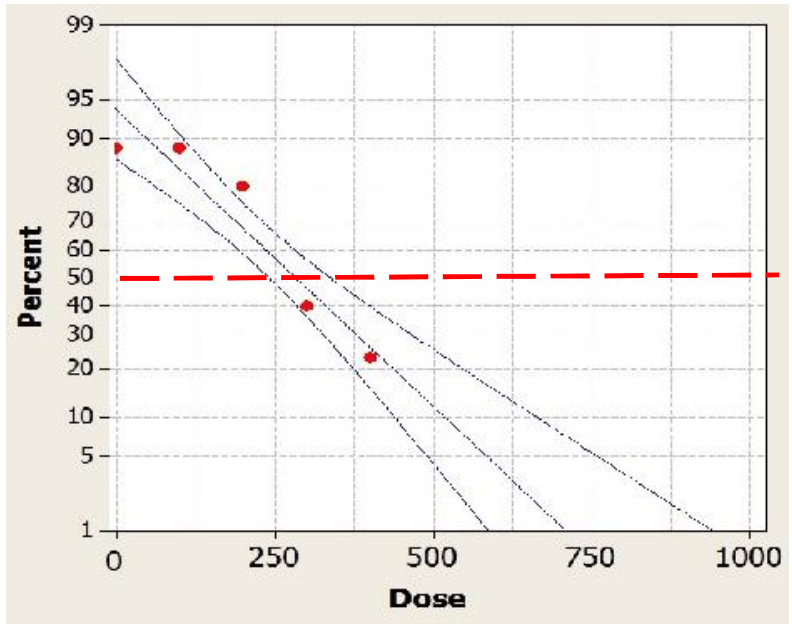

شكل r- تجزيه يروبيت براى تعيين دُز عامل كاهش دهنده •هـ\% بقا

Figure 2. Probit analysis to determine of the dose that caused 50\% reduction survival 


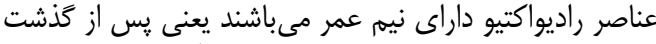

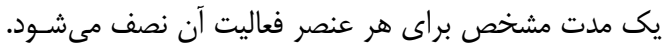

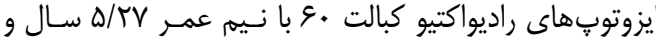

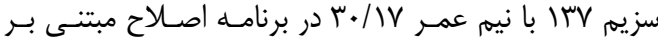

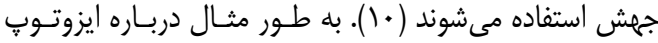

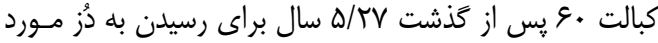

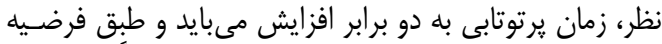

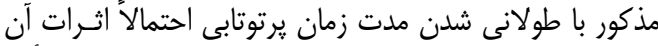

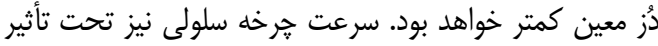

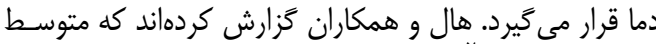

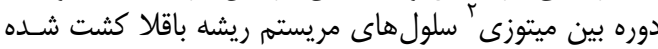

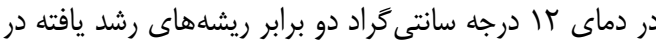

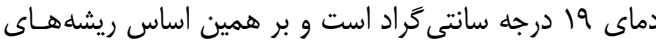

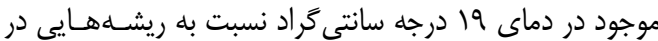

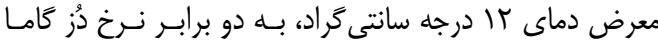

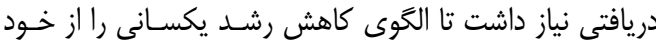

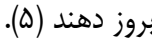

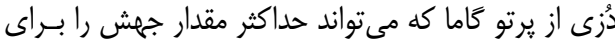

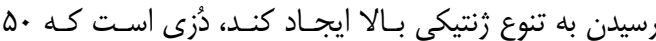

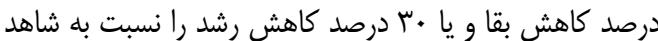

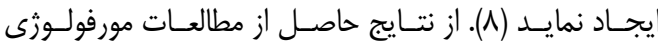

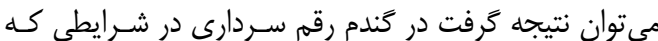

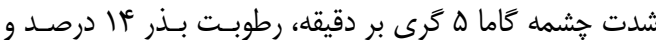

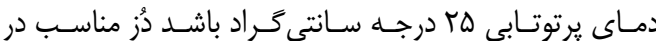

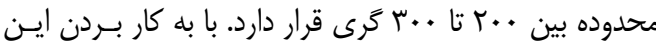

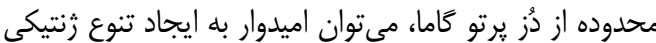

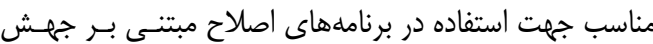

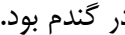

$$
\text { تشكر و قدردانى }
$$

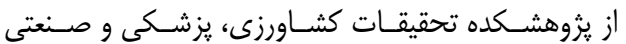

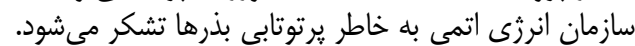

تعيين دُز مناسب يرتوتابى اولين كام در آغـاز يـــ برنامـهـ

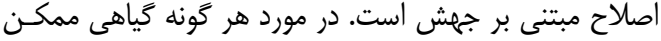

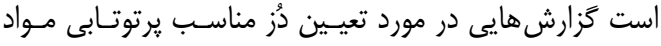

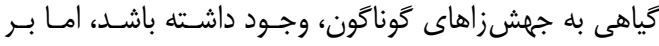

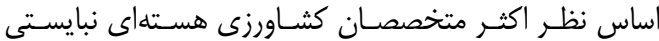

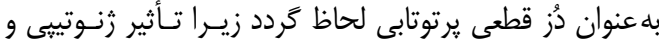

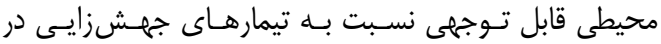

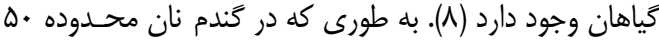

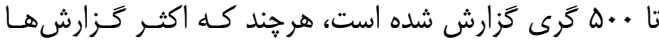

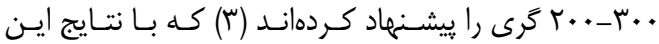

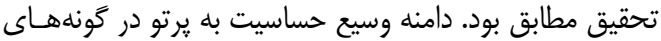

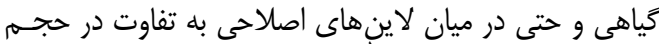

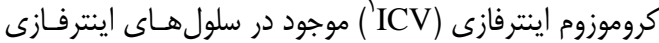

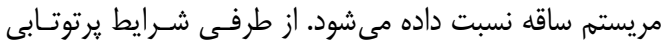

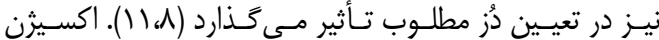

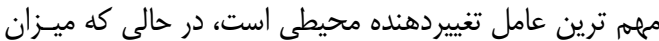

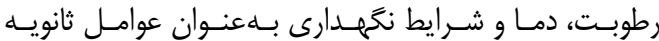

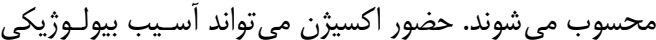

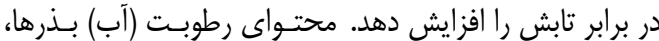

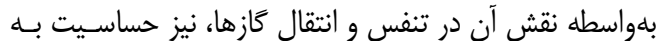

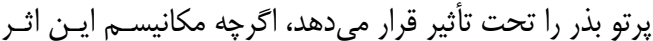

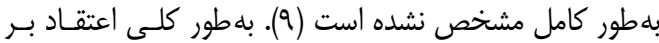

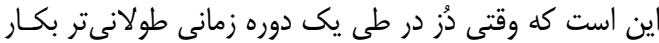

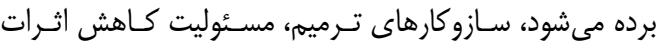

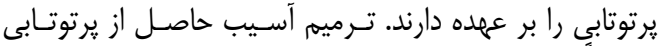

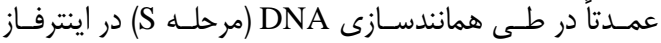

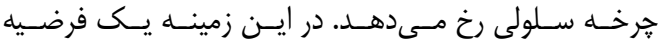

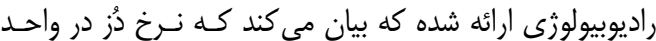

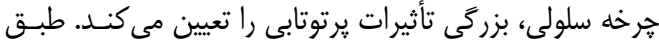

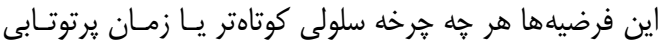

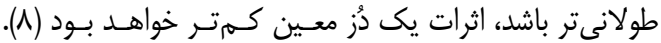

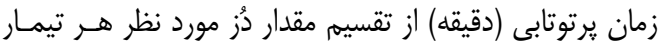

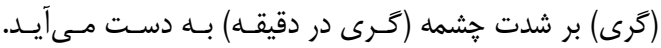


1. Borzouei, M. H. Kafi, B Khazaei, M. Naseriyan and A Majdabadi. 2010. Effects of gamma radia on germination and physiological aspects of wheat (Triticum aestivum L.) Seedlings. Pakestan Journal Botany, 42(4): 2281-2290.

2. Chaudhuri, K.S. 2002. A simple and reliable method to detect gamma irradiated lentil (Lens culinaris Medik.) seeds by germination efficiency and seedling growth test. Radiation Physics and Chemistry, 64(2): 131-136.

3. FAO/IAEA Database of Mutant Variety and Genetic Stock: http://mvgs.iaea.org.

4. FAO; Food and Agriculture Organization, Available http://faostat.fao.org. Last accessed 1 October 2016.

5. Hall, E.J., R. Oliver, B.J. Shepstone and J.S. Bedford. 2003. On the population kinetics of the root meristem of Vicia faba exposed to continuous irradiation. Radiation Research, 27(4): 597-603.

6. Kia, M., N.A. Babaeian Jelodar and N.A. Bagheri. 2010. Study on Salt Tolerance of Gamma Ray Induced Mutants in 032 Soybean Cultivar in Greenhouse Condition. Journal of Crop Breeding, 2(5): 47-56 (In Persian).

7. Kiong, A.L.P., A.G. Lai, S, Hussein and A.R. Harun. 2008. Physiological responses of Orthosiphon stamineus plantlets to gamma irradiation. American-Eurasian Journal of Sustainable Agriculture, 2(2): $135-149$.

8. Kodym, A., R. Afza, B.P. Forster, Y. Ukai, H. Nakagawa and C. Mba. 2012. Methodology for physical and chemical mutagenic treatments. In: Shu, Q.Y., B.P. Forster and H. Nakagawa (eds.) Plant mutation breeding and biotechnology. 169-180 pp., CABI, Oxford, UK.

9. Majd, F. and M.R. Ardakani. 2010. Nuclear techniques in agriculture sciences, $2^{\text {nd }}$ edn. Univerity of Tehran Press, Tehran, Iran, 381 pp (In Persian).

10. Mba, C. and Q.Y. Shu. 2012. Gamma irradiation. In: Shu, Q.Y., B.P. Forster and H. Nakagawa (eds.) Plant mutation breeding and biotechnology. 91-98 pp., CABI, Oxford, UK.

11. Mousavi Shalmani, A.M., B. Naserian Khiabani, H. Ahari Mostafavi. M. Heidarzadeh. and A. Majdabadi. 2009. Nuclear agriculture (from science to practical aspect). 1st edn. Nuclear Science and Technology Institute, Karj, Iran, 518 pp (In Persian).

12. Nakagawa, H. 2009. Induced mutations in plant breeding and biological researches in Japan. Crops, 242(188): 48-54

13. Preussa, S.B. and A.B. Britta. 2003. A DNA-damage-induced cell cycle checkpoint in Arabidopsis. Genetics, 164(1): 323-34.

14. Samadi Gorji, M., A. Zaman Mirabadi, V. Rammeah, aliollah, M. Hasanpour and A. Esmailifar. 2015. Evaluation of Agronomic Traits of Mutants Induced by Gamma Irradiation in PF and RGS003 Varieties of Rapeseed (Brassica napus L.). Journal of Crop Breeding, 7(15): 135-144 (In Persian).

15. Sikder, S., P. Biswas, P. Hazra, S. Akhtar, A. Chattopadhyay, A.M. Badigannavar and S.F. D'Souza. 2013. Induction of mutation in tomato (Solanum lycopersicum L.) by gamma irradiation and EMS. Indian Journal of Genetics and Plant Breeding, 73(4): 392-399.

16. Singh, N. and H. Balyan. 2009. Induced mutations in bread wheat (Triticum aestivum L.) CV.' Kharchia 65" for reduced plant height and improve grain quality traits. Advances in Biological Research, 3(5-6): 215-221.

17. van Harten, A.M. 1998. Mutation breeding: theory and practical applications. 1st edn. Cambridge University Press, Uk, 338 pp.

18. Wi, S.G., B.Y. Chung, J.S. Kim, J.H. Kim, M.H. Baek, J.W. Lee and Y.S. Kim. 2007. Effects of gamma irradiation on morphological changes and biological responses in plants. Micron, 38(6): 553564. 


\title{
Probit Analysis to Determine of the Most Appropriate Dose of Gamma Irradiation in Wheat Mutation Breeding
}

\author{
Saeed Bagherikia ${ }^{1}$, Mohammad Hadi Pahlevani ${ }^{2}$, Ahad Yamchi ${ }^{3}$ and Khalil Zenalinezhad ${ }^{3}$ \\ 1- Ph.D. Student, Gorgan University of Agricultural Sciences and Natural Resources, \\ (Corresponding author: s.bagherikia@gau.ac.ir) \\ 2 and 3- Associate Professor and Assistant Professor, Gorgan University of Agricultural Sciences and Natural \\ Resources \\ Receive: December 25, 2016 Accepted: April 29, 2017
}

\begin{abstract}
Identification of the appropriate dose for mutagen material is the most important step in setting experiment and creating mutant genetic material. According to a definition the most appropriate dose of mutagen is a dose that causing a reduction of $50 \%$ survival or $30 \%$ growth, compared to control. The aim of this study was to evaluate the effect of different doses of gamma radiation ( 0 as a control, 100, 200, 300 and 400 Gary) on the early growth characteristics for determining the appropriate dose of gamma irradiation in wheat cultivar Sardari. Therefore, an experiment was conducted based on completely randomized design with three replications at the laboratory and greenhouse. Data analysis of seed germination experiment showed significant difference in all traits including rootlet length, stemlet length, fresh weight of rootlet, fresh weight of stemlet, dry weight of rootlet and dry weight of stemlet, the exception of germination rate. Analysis of data obtained from a greenhouse, also showed significant difference in plant height and survival percentage. Based on the best fitted regression model and probit analysis determined a dose causing reduction of $50 \%$ survival or $30 \%$ growth, compared to control. It was in the range between 200 to 300 Gary, for Sardari cultivar. It could help to create adequate genetic diversity by applying this range of gamma radiation, for using in the wheat mutation breeding programs.
\end{abstract}

Keywords: Cobalt 60, Gamma Irradiation, Genetic Diversity, Mutation Breeding, Regression Model, Wheat 